\title{
Práticas no parto: análise do cuidado em uma maternidade paraibana
}

RESUMO | Objetivo: Caracterizar as práticas em saúde no período do parto em uma maternidade Paraibana. Método: Pesquisa de origem exploratória, descritiva e quantitativa. O estudo ocorreu em 2018, em uma Maternidade Municipal, localizada no município de Cabedelo, Paraíba. A amostra se deu com a participação de 12 pacientes, que se encontravam no período puerperal. Os dados foram analisados sob estatística descritiva e organizados em duas tabelas. Foi aprovado pelo comitê de ética em pesquisa do Centro Universitário de João Pessoa sob n CAAE 83170617.8.0000.5176. Resultados: Ocorreu aplicação de ocitocina de rotina; Manobras desnecessárias; Amamentação na primeira hora de vida prejudicada e clampeamento precoce do cordão. Conclusão: Percebe-se a necessidade de investir-se em capacitações para os profissionais, visando quebrar a assistência inadequada e fragilizada que está sendo oferecida para as mulheres, no qual é rodeada de intervenções e cuidados desnecessários ao binômio.

Descritores: Assistência ao Parto; Parto Normal; Enfermagem Obstétrica.

\begin{abstract}
Objective: To characterize health practices during childbirth in a Paraíba maternity hospital. Method: Exploratory, descriptive and quantitative research. The study took place in 2018, in a Municipal Maternity, located in the city of Cabedelo, Paraíba. The sample took place with the participation of 12 patients, who were in the puerperal period. Data were analyzed using descriptive statistics and organized into two tables. It was approved by the research ethics committee of the Centro Universitário de João Pessoa under number CAAE 83170617.80000.5176. Results: Routine application of oxytocin occurred; Unnecessary maneuvers; Impaired breastfeeding in the first hour of life and early cord clamping. Conclusion: It is perceived the need to invest in training for professionals, aiming to break the inadequate and weakened care that is being offered to women, which is surrounded by interventions and unnecessary care for the binomial.
\end{abstract}

Keywords: Midwifery; Natural Childbirth; Obstetric Nursing.

RESUMEN | Objetivo: Caracterizar las prácticas de salud durante el parto en una maternidad de Paraíba. Método: Investigación exploratoria, descriptiva y cuantitativa. El estudio se realizó en 2018, en una Maternidad Municipal, ubicada en la ciudad de Cabedelo, Paraíba. La muestra se realizó con la participación de 12 pacientes, que se encontraban en el puerperio. Los datos se analizaron mediante estadística descriptiva y se organizaron en dos tablas. Fue aprobado por el comité de ética en investigación del Centro Universitário de João Pessoa con el número CAAE 83170617.80000.5176. Resultados: se produjo la aplicación de rutina de oxitocina; Maniobras innecesarias; Lactancia materna deteriorada en la primera hora de vida y pinzamiento temprano del cordón. Conclusión: Se percibe la necesidad de invertir en la formación de los profesionales, con el objetivo de romper con la atención inadecuada y debilitada que se ofrece a las mujeres, que está rodeada de intervenciones y cuidados innecesarios para el binomio.

Palabras claves: Asistencia de entrega; Parto normal; Enfermería obstétrica.

\section{Angélica Helena Tito Fernandes}

Enfermeira. Centro Universitário de João Pessoa.

ORCID ID: 0000-0002-5035-5593

\section{Alane Barreto de Almeida Leôncio \\ Enfermeira. Mestre em Enfermagem pelo Programa de Pós-Graduação em Enferma- gem da Universidade Federal da Paraíba (PPGENF/UFPB). Especialista em Saúde da Criança pelo Programa de Residência Multi- profissional do Estado da Paraíba. Docente do curso de enfermagem do Centro Univer- sitário de João Pessoa \\ ORCID ID: 0000-0003-4575-1900}

\section{Wilma Ferreira Guedes Rodrigues}

Enfermeira. Doutora em Enfermagem pela Universidade Federal do Rio Grande do
Norte. Docente do curso de enfermagem do Centro Universitário de João Pessoa ORCID ID: 0000-0002-9003-4807

\section{Luanna Silva Braga}

Enfermeira. Mestre em Enfermagem pelo Programa de Pós-Graduação em Enfermagem da Universidade Federal da Paraíba (PPGENF/UFPB). Docente do curso de enfermagem do Centro Universitário de João Pessoa

ORCID ID: 0000-0002-009-0406

\section{Jeferson Barbosa Silva}

Enfermeiro. Doutor em Enfermagem pelo Programa de Pós-Graduação em Enfermagem da Universidade Federal da Paraíba (PPGENF/UFPB).

ORCID ID: 0000-0002-1083-1305
Anna Claudia Silva de Araujo

Enfermeira. Mestre em Biotecnologia e inovação em Saúde.

ORCID ID: 0000-0002-7608-3979

Recebido em: 25/11/2021

Aprovado em: 15/12/2021

INTRODUÇÃO

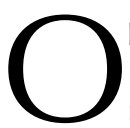
parto é um acontecimento feminino e natural onde é chegado o momento desejado pela mulher, o nascimento do bebê. No passado o parto acontecia em ambiente domiciliar, em que a parturiente normalmente era observada por uma parteira de confiança possuindo conhecimentos por tradição oral e tendo a presença de seus familiares. No 
Brasil, a demanda do desenvolvimento da assistência do parto domiciliar sendo parteiras tradicionais esta com intensa abordada desde década de 1970 (1).

Os modelos hegemônicos têm grande mudança na vida da mulher tirando o empoderamento e tornando máquina e o seu bebê o produto. O hospital, por sua vez, torna-se a fábrica, o corpo da mãe a máquina e o bebê representa o produto de um processo de fabricação industrial(2).

A chegada da institucionalização e dos médicos mudando o cenário do parto deixando robótico e técnico o procedimento causando o aumento de número de procedimento indesejado. O modelo de parto no Brasil esta resistente e centralizado pelo profissional médico(2).

A prática de humanização na assistência a parturiente no método de parir mostra uma atenção apontada à mulher em particular, sendo específicas, e assuntos biológicas, condições sociais, éticas, educacionais e psíquicas(3). No estudo de Carvalho(4) as mulheres são submetida a rotina de intervenções desnecessárias sem ser informada sobre as práticas que séria utilizadas.

Sem essas informações, a assistência pode está sendo negligenciada pela equipe causando desumanização as mulheres. Nessa visão, a assistência na parição no Brasil é uma maneira de violência às mulheres desumanizada, exposta a prática rotineira da episioto$\operatorname{mia}(5)$.

As intervenções desnecessárias no parto podem causar uma mudança no emocional, trauma psicológico e físico e que cada mulher tenha um cuidado individual. A perspectiva do parto para melhorar o bem-estar e emocional a dor extrema é um resultado de choque psicológico para algumas, para outras os efeitos indesejado dos fármacos sendo influência negativa na experiência do nascimento(6).

As práticas úteis são condutas dos profissionais de enfermagem e da área
A prática de
humanização na assistência a parturiente no método de parir mostra uma atenção apontada à mulher em particular, sendo específicas, e assuntos biológicas, condições sociais, éticas, educacionais e psíquicas(3). No estudo de Carvalho(4) as mulheres são submetida a rotina de intervenções desnecessárias sem ser informada sobre as práticas que séria utilizadas de saúde para ser utilizadas, para mudar o cenário de atenção ao parto. Proporcionar aos profissionais enfermeiros e médicos, maior conhecimento no atendimento da mulher, podendo aumentar a segurança dessa parturiente, no acontecimento tão importante de sua vida(7). As leituras com atenção indicam que ainda há ausências no entendimento das qualidades e das limitações no processo de trabalho dos profissionais que atuam na atenção ao parto, com baixa adesão às boas práticas obstétricas(8).

A necessidade dos profissionais de enfermagem em ter uma postura em cuidar das usuárias com humanização, dignidade e valorização. As usuárias dos serviços de saúde, não reclamam do conhecimento tecnológico no seu atendimento, mas a falta de interesse e de responsabilização do serviço em torno de si e de seu problema encontrado(9).

Desse modo, tem-se por questão de pesquisa: Como se dá as práticas no parto em uma maternidade no Estado da Paraíba? O objetivo do estudo, foi caracterizar as práticas em saúde no período do parto em uma maternidade Paraibana.

\section{MÉTODO}

Pesquisa de origem exploratória, descritiva e quantitativa. O estudo ocorreu em 2018, em uma Maternidade Municipal, localizada no município de Cabedelo, Paraíba. A amostra se deu com a participação de 12 pacientes, que se encontravam no período puerperal, mediante critérios de inclusão e exclusão. Foram elencados como critérios de inclusão: possuir idade superior a 18 anos; estar alocada na maternidade escolhida, ter vivenciado o parto normal e aceitar participar da pesquisa assinando o Termo de Consentimento Livre e Esclarecido (TCLE). Foram excluídas, as mulheres que pariram em outras modalidades de parto, com ida- 
de inferior a 18 anos e que foram admitidas após o período da pesquisa.

Foi utilizado como recurso, um instrumento relacionado as práticas no parto. Este instrumento foi construído pelos autores desta pesquisa. Os dados analisados foram submetidos estatística descritiva, utilizando o Microsoft Excel. Mediante a interpretação dos resultados, estes foram fundamentados com estudos relacionados a temática. A pesquisa seguiu a Resolução 466/120(10) e foi aprovada pelo Comitê de Ética em Pesquisa do Centro Universitário de João Pessoa sob número CAAE 83170617.8.0000.5176.

\section{RESULTADOS}

Abaixo, ocorreu a descrição dos resultados, no qual foram organizados em duas tabelas. A primeira sistematiza quanto as intervenções obstétricas, e a segunda acerca da amamentação e outras práticas relacionadas.

Na variável negaram ou não ofereceram métodos de alivio da dor observa-se que $(n=25 \%)$ responderam que sim. Em relação a variável administraram ocitocina de rotina no trabalho de parto quase a metade das entrevistadas $(\mathrm{n}=41 \%$ ) responderam que sim. Quanto a variável realizaram manobra de Kristeller no parto um número considerável de mulheres $(n=41 \%)$ responderam que sim. E acerca da variável realizaram episiotomia algumas entrevistadas $(n=33 \%)$ responderam que sim.

A segunda tabela, refere-se à amamentação, agendamento de cesárea eletiva e clampeamento do cordão de forma precoce, no qual foram identificadas algumas intervenções desnecessárias.

Com relação a Tabela 4, a variável retardaram ou impediram o contato do bebê com a mãe após o parto, impediram o aleitamento materno na primeira hora de vida do recém-nascido e agendaram cesárea sem indicação clínica foi constatado que houve esse tipo de prática $(n=08 \%)$. Acerca da variável foi realizado clampeamento precoce do cordão umbilical a maioria das mulheres relataram que $\operatorname{sim}(n=58 \%)$.

\section{DISCUSSÃO}

A menor prevalência de boas práticas foi nas regiões Norte e Nordeste, territórios menos avançados no país. A repetição de algumas intervenções foi menor, o que não significa absolutamente a um padrão pouco intervencionista e mais "natural" (11).

Conforme este manual(12), algumas práticas são reconhecidas como favoráveis para o trabalho de parto nas mulheres como a alimentação, mobilidade e o uso de técnicas não farmacológicos para alívio da dor. No estudo de Leal(11), as intervenções médicas foram consideradas exageradas no trabalho de parto e no parto, tendo apenas $5,6 \%$ das parturientes de risco habitual e $3,2 \%$ das primíparas com um parto normal sem nenhuma intervenção no processo do trabalho de parto.

A infusão de ocitocina de rotina é uma técnica muito usada para acelerar o trabalho de parto e costuma acontecer em $40 \%$ das parturientes de risco habitual, sendo mais frequente no setor público em mulheres com baixo nível de escolaridade corroborando com a presente pesquisa que obteve elevado número de mulheres que receberam a infusão de ocitocina de rotina (05; $41 \%$ ) no parto normal. É importante salientar que a OMS considera que a administração de ocitocina de rotina no trabalho de parto é uma prática prejudicial ou ineficaz e deve ser utilizada

Tabela 1 - Distribuição das variáveis relacionadas a prática de intervenções obstétricas. Cabedelo, Paraíba, 2018 (N = 12).

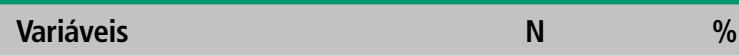

Negaram ou não foi oferecido métodos de alívio da dor

$\begin{array}{lll}\text { Sim } & 03 & 25 \% \\ \text { Não } & 09 & 75 \%\end{array}$

Negaram ou não foi oferecido líquidos e alimentos no trabatho de parto

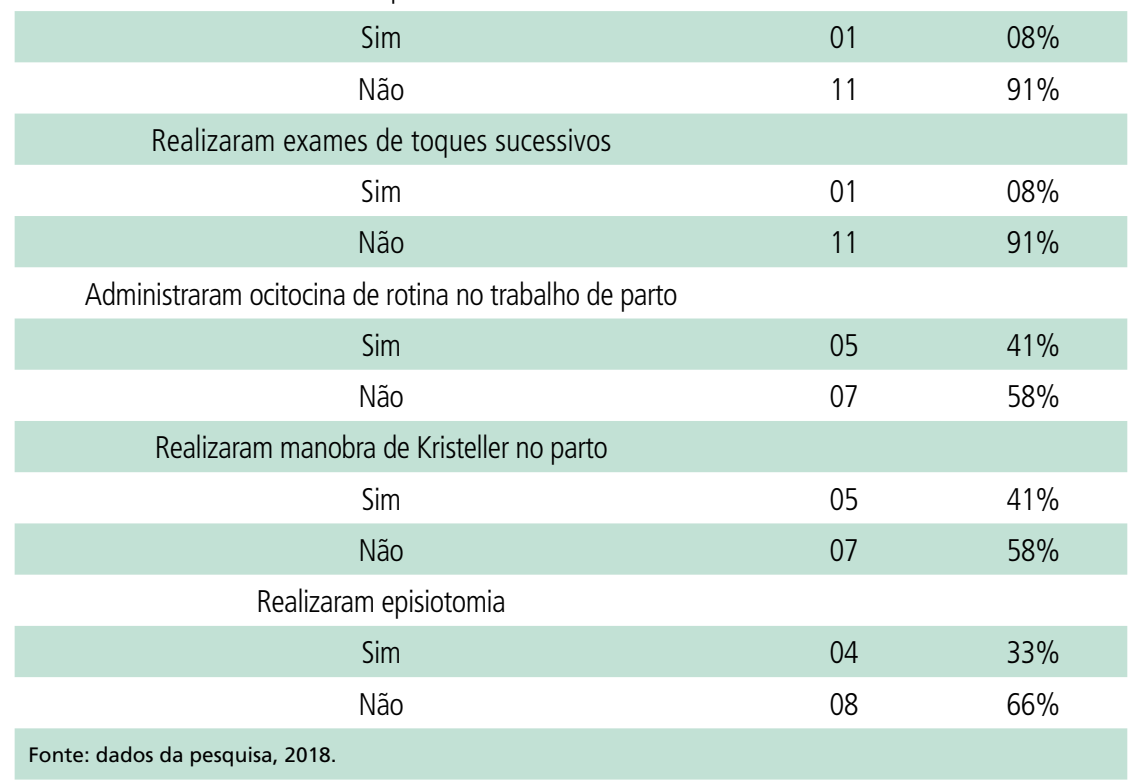


apenas em real necessidade(11).

A manobra de Kristeller nos partos vaginais tem alta taxa de prevalência ocorrendo em cerca de $37 \%$ das mulheres de baixo nível escolar corroborando com o que foi constatado no referido estudo no qual a manobra de Kristeller foi realizada em $41 \%$ dos partos(11). É importante lembrar que a manobra de Kristeller é um procedimento proscrito no parto, conforme orienta o (MS) na diretriz de Assistência ao Parto Normal(12), proibindo a realização de tal manobra no segundo estágio do parto pela equipe obstétrica.

No artigo de Carvalho(4), a prática da episiotomia foi inserida à rotina na assistência obstétrica sem que existissem evidências científicas que comprovasse seus riscos e benefícios de ser realizada. A episiotomia é definida como uma incisão cirúrgica cometida no segundo estágio do TP, no começo da expulsão do feto(4), contudo traz em sua história ao longo da obstetrícia informação para sua prática quando a perigo de danos no assoalho pélvico, possível ocorrência de distorcias genitais, tal como, sofrimento fetal e materno(13).

Consequentemente realizada dentro dos critérios recomendados pela OMS, Figueiredo et al(14); Silva et al. (15) apontam como relacionada pela prática da episiotomia, à capacidade no aumento de perda sanguínea, à inflamação, disfunção sexual, a dispareunia, incontinência urinária e prolapso do colo do útero; e, consequências físicas e psicológicas.

As Diretrizes Nacionais de Assistência ao Parto Normal orientam acerca da atuação fisiológica no terceiro estágio do parto abrangendo um complexo de cuidados que inclui a assistência ao clampeamento tardio do cordão umbilical, apenas, após parar a pulsação. Esse estudo constatou que o clampeamento precoce do cordão umbilical foi realizado em $(n=58 \%)$ dos $\mathrm{RN}$, privando-os dos benefícios de um clampea-

Tabela 2 - Distribuição das variáveis acerca dos bebês e amamentação. Cabedelo, Paraiba, 2018 ( $=12)$.

$$
\text { Variáveis }
$$

N

$\%$

\begin{tabular}{|c|c|c|}
\hline \multicolumn{3}{|c|}{$\begin{array}{l}\text { Retardaram ou impediram o contato do bebê com a mãe após } \\
\text { o parto }\end{array}$} \\
\hline Sim & 01 & $08 \%$ \\
\hline Não & 11 & $91 \%$ \\
\hline
\end{tabular}

Impediram o aleitamento materno na primeira hora de vida do recém-nascido

\begin{tabular}{|ccc|}
\hline \multicolumn{1}{|c|}{$\begin{array}{c}\text { Sim } \\
\text { Não }\end{array}$} & 01 & $08 \%$ \\
\hline Foi agendado cesárea sem indicação clínica & 11 & $91 \%$ \\
\hline Sim & & \\
\hline Não & 01 & $08 \%$ \\
\hline Foi realizado clampeamento precoce do cordão umbilical & 11 & $91 \%$ \\
\hline Sim & & \\
\hline Não & 07 & $58 \%$ \\
\hline Fonte: dados da pesquisa, 2018. & 05 & $41 \%$ \\
\hline
\end{tabular}

mento tardio(12).

A importância do clampeamento tardio (de 1 a 3 minutos após o nascimento) está correlacionada à concentração da hemoglobina e reservas elevadas de ferro nos seis primeiros meses de vida dos lactentes, além da prevenção de anemia até o primeiro ano de vida(16).

O clampeamento tardio do cordão umbilical tem benefícios para o $\mathrm{RN}$ pré-termo como circulação de transição, melhora nos níveis de glóbulos vermelhos, menor necessidade de transfusão de sangue, e menor incidência de enterocolite necrotizante e hemorragia intraventricular(17).

Mascola et al. (17), comenta que os obstetras, enfermeiros e outros prestadores de cuidados obstétricos que utilizam o clampeamento tardio do cordão umbilical no RN a termo devem monitorar a icterícia neonatal.

A mulher tem uma necessidade exclusiva sendo importante para um apoio e comunicação, observar as modificações de comportamento como sinais de estresse que necessita o método de alívio da dor sendo um suporte(16).

Conforme Silva et al. (18) o conforto deve ser transmitido com um olhar, uma escuta delicada, da percepção no momento do parto e sintonia do profissional de enfermagem com a parturiente.

É importante a inserção do parto e do nascimento domiciliar assistido por parteiras no Sistema Único Saúde, entre os métodos para o cuidado integral à saúde da mulher e da criança(1). O Ministério da Saúde tem capacitado o profissional com solução que respaldam a atenção ao parto pela Enfermagem, prática recente mostra a necessidade de desenvolvimento com políticas definidas que assegurem a gestante(19).

Com o parto domiciliar as mulheres têm o empoderamento de fazer a sua escolha do local do parto e posição que estiverem confortável e segura. Assim, os partos realizados nas diversas formas de posições verticais estão relacionados ao tempo reduzido do período expulsivo quando comparados aos partos em posição horizontal(18). 
O modelo hegemônico a mulher é colocada em posição horizontal que pode oferecer limitação durante o trabalho de parto as evidências mostrar que as realidades atuais existem muIheres usando a posição horizontal. Evidencia-se uma distância significativa entre o que é preconizado como prática e a realidade nos serviços de saúde(20).

Atenção no parto, aos exageros de intervenções causando uma prática de rotina uma delas é a episiotomia sem avaliar o risco e benefícios realizados. A prática da episiotomia é uma rotina desde o período anterior com objetivo de diminuir o prejuízo ocasionado pela laceração do períneo, reduzir o dano de incontinência urinária, fecal e ajudar o recém-nascido do choque(11). Muitas mulheres devem saber que têm o direito de pode procurar ajuda estando respaldada pela lei e conhecimento. Quando as mulheres procuram ajuda, estão também em busca de uma compreensão, pois para elas e suas famílias o momento da gravidez e do parto, em particular, é único na vida e carregado de fortes emoções(12).

\section{CONCLUSÃO}

O estudo alcançou os seus objetivos propostos inicialmente, caracterizando as práticas relacionadas ao parto na maternidade escolhida. Percebe-se a necessidade de investir-se em capacitações para os profissionais, visando quebrar a assistência inadequada e fragilizada que está sendo oferecida para as mulheres, no qual é rodeada de intervenções e cuidados desnecessários ao binômio.

Desde o pré-natal essas mulheres precisam ser orientadas, junto ao seu acompanhante, no qual precisa ser falado sobre os seus direitos no momento do parto, e além disso, abordar quanto a humanização, visando reduzir situações contraditórias para a assistência a parturiente. É relevante, que novas pesquisas sejam sintetizadas, na intenção de aprofundar do tema e qualificar os profissionais de saúde envolvidos.

\section{Referências}

1- Brasil, Ministério da Saúde. Caderno de Atenção Básica, Atenção ao pré -natal de baixo risco n³2. Brasília-DF 2013.

2- Sanfelice CFO. et al. Do Institucional ao parto Domiciliar. Revista Rene, p.363, 2014.

3- Nascimento JP. et al. 0 empoderamento da mulher no parto domiciliar planejado. Revista de Enfermagem, p.4183, novembro 2016.

4- Carvalho VF. et al. Práticas prejudiciais ao parto: relatos dos trabalhadores de Saúde de Sul do Brasil. Revista Rene, p. 93, 2010.

5- Oliveira TR. et al. Percepção das mulheres sobre violência obstétrica. Revista Enfer. UFPE on line. Recife, v 11, n.1, p.40-6, jan. 2017.

6- Brasil. Ministério da Saúde. Humanização do parto e nascimento. Caderno Humaniza SUS, v 4, Brasília-DF 2014.

7- Lopes CV. et al. Experiências vivenciadas pela mulher no momento do parto e nascimento de seu filho. Cogitare Enfermagem. julh/set 2009.

8- Carvalho EMP. et al. Adesão às boas práticas na atenção ao parto normal: construção e validação de instrumento. Rev. Esc. Enfermagem USP, Brasilia-DF, p. 891, 2015.

9- Santos LM. et al. Relacionamento entre profissionais de saúde e parturientes: um estudo com desenhos. Revista Enferm UFSM. 2011.

10- Brasil. Ministério da Saúde. Conselho Nacional de Saúde. Resolução $n^{\circ} 466 / 2012$. Aprovar diretrizes e normas regulamentadoras de pesquisas envolvendo seres humanos. 12. dezembro. 2012.

11- Leal MC. et al. Intervenções Obstétricas durante o trabalho de parto em mulheres brasileiras de risco habitual. Cad. Saúde Pública. Rio de Ja- neiro, vol.30 p. 22-4, 2014.

12- Brasil. Ministério da Saúde. Diretriz do Parto Normal, Versão resumida. Brasília-DF. 2017.

13- Costa NM. et al., Episiotomia nos partos normal: incidência e complicações literatura. Revista Facene/Famene, Mossoró/RN v.9, n. 2, p. 45-50, 2011.

14- Figueiredo GS. et al., Ocorrência de episiotomia em partos acompanhados por enfermeiros obstetras em ambiente hospitalar. Revista Enfermagem UERJ. Rio de Janeiro, v. 19, n. 2, p. 181-1852011 abr./jun 2011.

15- Silva NLS. et al. Dispareunia, dor perineal e cicatrização após episiotomia. Revista de Enfermagem UERJ, v. 21, n. 2, p. 216-20, 2012.

16- Steffen EL. et al. Efeito do pinçamento tardio do cordão umbilical nos níveis séricos de ferritina de crianças de 0, 3 e 6 meses de vida. Rev Inst Adolfo Lutz, São Paulo, 2012; 160-5.

17- Mascola MA, Porter TF, Chao TTM. Delayed umbilical cord clamping after birth. Committee Opinion No. 684. American College of Obstetricians and Gynecologists. Obstet Gynecol. January, p.5-10; 2017.

18- Silva LS. et al. Os saberes das mulheres acerca das diferentes posições de parir; uma contribuição para o cuidar. Revista enfermagem UFPE, p.3532-5, setembro. 2016.

19- Mattos DV. et al. 0 Enfermeiro obstetra no parto domiciliar planejado. Revista de Enfermagem UFPE on line, Recife v 10, n. 2, 568-75, fev., 2016.

20- Amorim MMR, Souza ASR, Porto, AMF. Indicação de cesariana baseada em evidências: parte I. Revista Femina, 2010. 